\title{
Absolute kinematics of radio source components in the complete S5 polar cap sample
}

\section{First and second epoch maps at $8.4 \mathrm{GHz}$}

\author{
E. Ros ${ }^{1}$, J. M. Marcaide ${ }^{2}$, J. C. Guirado ${ }^{2,3}$, and M. A. Pérez-Torres ${ }^{4}$ \\ 1 Max-Planck-Institut für Radioastronomie, Auf dem Hügel 69, 53121 Bonn, Germany \\ 2 Departament d'Astronomia i Astrofísica, Universitat de València, 46100 Burjassot, València, Spain \\ 3 Observatorio Astronómico, Universitat de València, 46100 Burjassot, València, Spain \\ 4 Istituto di Radioastronomia, Via Gobetti 101, 40129 Bologna, Italy
}

Received 20 April 2001 / Accepted 4 July 2001

\begin{abstract}
We observed the thirteen extragalactic radio sources of the S5 polar cap sample at $8.4 \mathrm{GHz}$ with the Very Long Baseline Array, on 1997.93 and 1999.41. We present the maps from those two epochs and briefly discuss the morphological changes experimented by some of the radio sources in the $1.4 \mathrm{yr}$ elapsed. These results correspond to the first two epochs at $8.4 \mathrm{GHz}$ of a program directed to study the absolute kinematics of the radio source components of the members of the sample by means of phase delay astrometry at $8.4,15$ and $43 \mathrm{GHz}$.
\end{abstract}

Key words. astrometry - techniques: interferometric - quasars: general - BL Lacertae objects: general

\section{Introduction}

Very-long-baseline interferometry (VLBI) allows routine imaging of compact radio sources with milliarcsecs resolution. Use of phase-delay astrometric techniques in VLBI also allows determinations of relative positions with nearly microarcsecs accuracy (e.g., Marcaide \& Shapiro 1983). Moreover, phase delay astrometry, unlike group-delay astrometry, permits a reliable subtraction of the source structure contribution to the delay, by referring such contribution to a reference point in the map. Such a (fixed) reference point provides an unambiguous identification of radio source features seen at different epochs, crucial for the determination of the absolute kinematics of the components (see Charlot et al. 1990; Guirado et al. 1995b; Ros et al. 1999).

In the past, our group has successfully applied phasedelay astrometry techniques to a number of pairs of radio sources. Guirado et al. $(1995,1998)$ studied the pair QSO $1928+738 / \mathrm{BL} 2007+777$ (about $5^{\circ}$ apart) at 2.3, 5, and $8.4 \mathrm{GHz}$. Ros et al. (1999) added a new source

Send offprint requests to: E. Ros,

e-mail: ros@mpifr-bonn.mpg.de
(BL 1803+784) to this pair, and extended the phaseconnection technique (Shapiro et al. 1979) to separations of 6.8 from observations at $8.4 \mathrm{GHz}$. Pérez-Torres et al. (2000) successfully applied the phase-connection technique to the radio sources QSO $1150+812 / \mathrm{BL} 1803+784$ (nearly $15^{\circ}$ apart), from observations at 2.3 and $8.4 \mathrm{GHz}$. In addition, Ros et al. (2000) and Pérez-Torres et al. (2000) have demonstrated that dual-frequency observations are not required anymore to subtract the ionospheric delay from the data. Indeed, those authors showed that it is possible to accurately model the ionosphere contribution from Global Positioning System data taken at sites nearby VLBI stations.

The astrometric results mentioned above have encouraged us to try to demonstrate the feasibility of the phase-connection technique for a complete sample of radio sources, using single-frequency VLBI observations, with the aim of studying the absolute kinematics of all the sources in the sample. Our sample consists of the 13 sources selected by Eckart et al. (1986, 1987) from the S5 survey (Kühr et al. 1981), with the following selection criteria: a) declination $\delta \geq 70^{\circ}$, galactic latitude $\left|b_{\mathrm{II}}\right| \geq$ $10^{\circ}$; b) flux density $S_{5 \mathrm{GHz}} \geq 1$ Jy at the epoch of the 
survey; and c) spectral index $\alpha_{2.7,5 \mathrm{GHz}} \geq-0.5(S \sim$ $\left.\nu^{+\alpha}\right)$. Throughout the paper, we will refer to those 13 radio sources as "the complete S5 polar cap sample".

We show in Fig. 1 the sky distribution of the members of the complete S5 polar cap sample, and indicate their names and relative angular separations. All 13 members of this sample are at such close angular distances that the use of an appropriate observing scheme permits a successful phase-connection of the data for a 24-hour observing Very Long Baseline Array (VLBA) run. Provided that enough phase-delay data (usually several hours) are acquired for each of the 13 radio sources, we should be able to solve for their positions with accuracies better than 0.1 milliarcsecs (mas). Once we precisely determine the relative positions of all the members of the S5 polar cap sample at $8.4 \mathrm{GHz}$ for several epochs, the registration of the maps should be straightforward.

Such an astrometric analysis should allow us to unambiguously discern - for each of the 13 radio sources which components are moving and which are stationary. Finding stationary components in 15 sources (the 13 of our sample, 3C 345, Bartel et al. 1986; and 1038+528 A, Marcaide et al. 1994) would strongly support the standard model for AGNs (Blandford \& Königl 1979). On the contrary, finding that some have no stationary components might reveal phenomena unthought. Knowing which components move and how, in an absolute sense, should allow us to propose the correct physical models.

As a first step in the astrometric process, we present here the hybrid maps of all radio sources at $8.4 \mathrm{GHz}$ for the two observing epochs. The astrometric results will be shown in further publications.

We concisely describe the $8.4 \mathrm{GHz}$ observations in Sect. 2, and present the source maps corresponding to the first (1997.93) and second (1999.41) epochs for all 13 radio sources in Sect. 3. We conclude with some summary remarks in Sect. 4.

\section{Observations}

We observed the complete S5 polar cap sample at $8.4 \mathrm{GHz}$ on 6 December 1997 (epoch 1997.93) and on 28 May 1999 (epoch 1999.41) with the VLBA, each time for 24 hours. We used each case the recording mode 128-8-1 (1 bit sampling, 16 channels per each of the $8 \mathrm{IFs}$, with a total of 128 channels), yielding a total bandwidth of $64 \mathrm{MHz}$, in right circular polarization. Data were correlated at the VLBA Array Operations Center of the National Radio Astronomy Observatory (NRAO) in Socorro, New Mexico, using a basic integration time of $4 \mathrm{~s}$. All radio sources were detected and provided fringes for all baselines. We observed in groups of 3 or 4 radio sources in a cyclic way with duty cycles of about $6 \mathrm{~min}$. Every scan was $78 \mathrm{~s}$ long, and the small (a few seconds) time gap between different scans was used for slewing the VLBA antennas. We

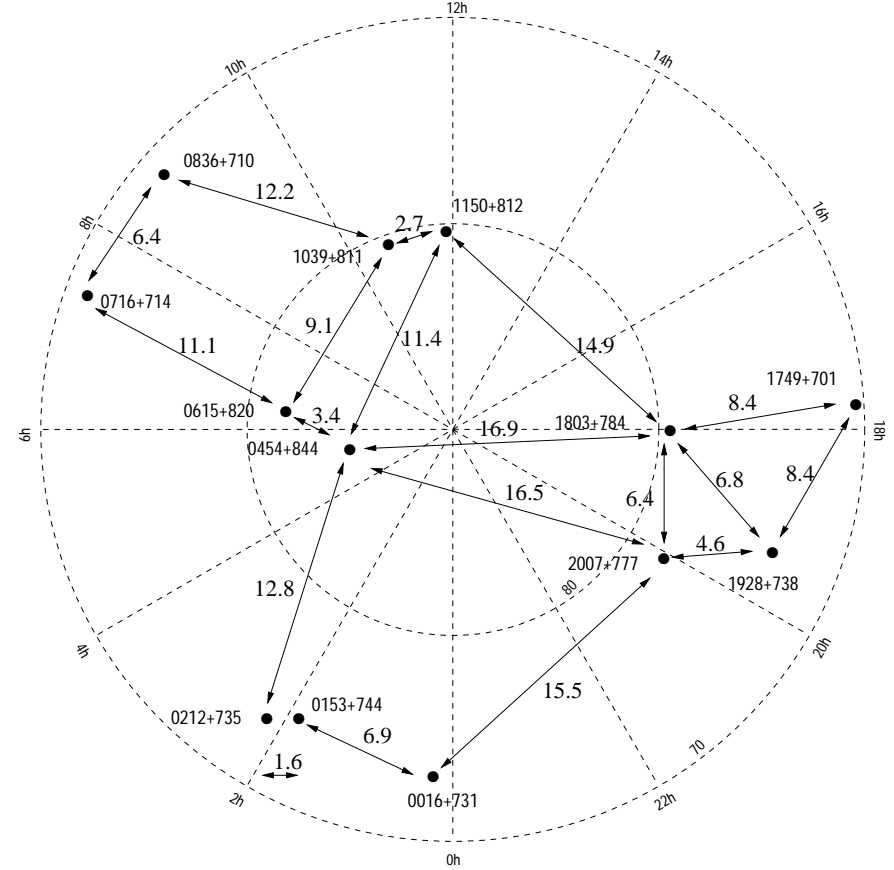

Fig. 1. Distribution of the complete S5 polar cap sample in the northern sky, centered at the celestial north pole. The black dots represent the positions of radio sources, and the angular distances among them are indicated in arc degrees with arrows.

replaced one or two members of the group of observed sources by new members about every 2 hours, until all 13 members of the sample were observed. This observing scheme resulted in a time coverage for each single radio source of about 4 hours. We tracked the clock behavior of relevance for the ulterior astrometric analysis - by including some scans of BL $0454+844$ in all groups observed during the first 12 hours, and of BL $2007+777$ in all groups observed during the second 12 hours.

After having fringe-fitted the correlator output, we obtained correlation amplitudes for each source. We then constructed the visibility amplitudes in a standard manner, using the antenna gain and system temperature information provided by each station. We exported the data for use with the Caltech VLBI Package (Pearson 1991) and the difference mapping software DIFMAP (Shepherd et al. 1994).

\section{Imaging results}

We obtained images of all 13 radio sources using standard hybrid mapping techniques. We found only minor calibration problems for data from the North Liberty antenna at epoch 1997.93; we had to multiply gain by a factor 2.03 - for all IF channels - to get consistent results with the other nine antennas. We show the maps obtained in Figs. 2 through 16. In all figures, east is left, and north is up. We list the main parameters of those maps in Table 1 . We use 

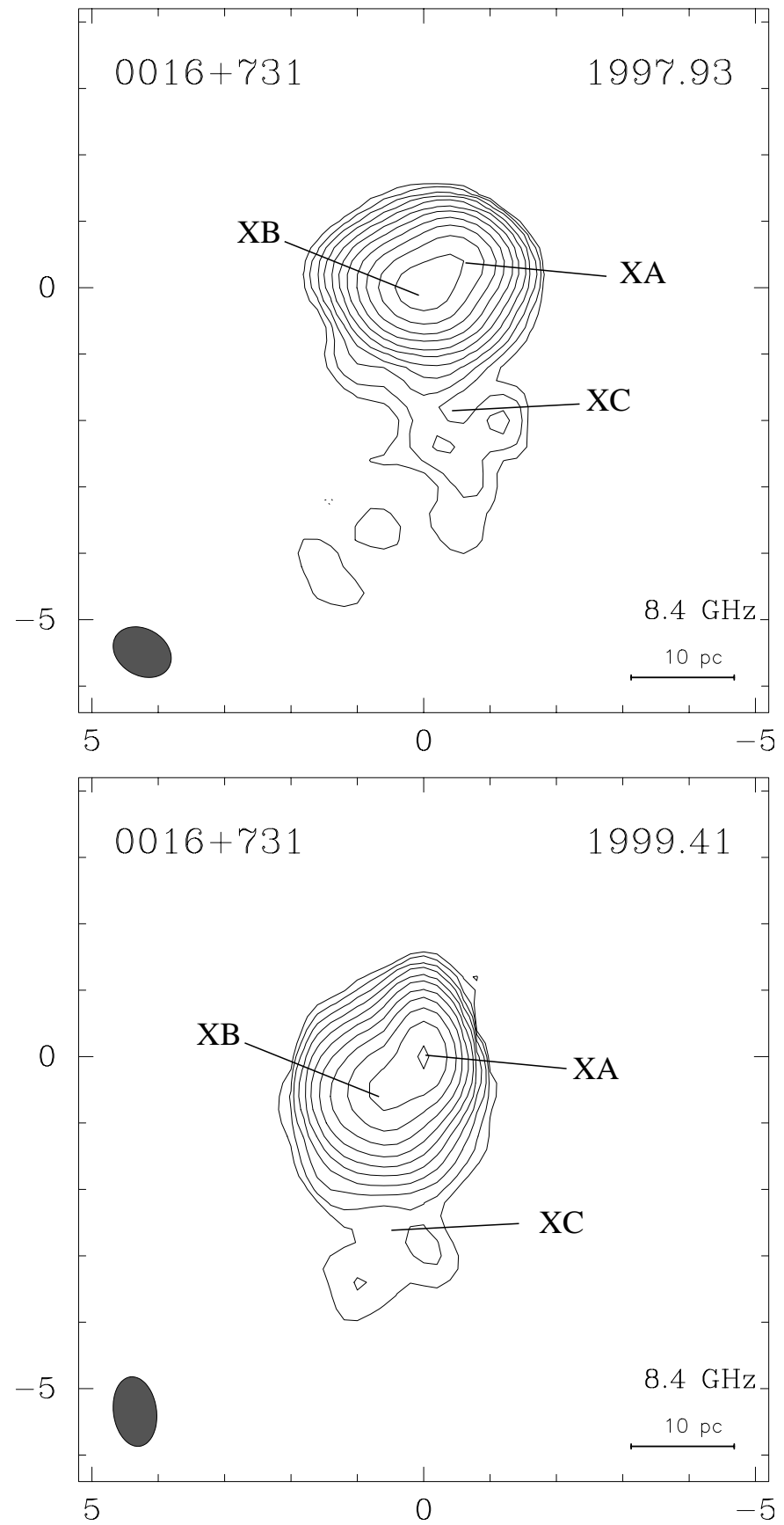

Fig. 2. VLBA images of QSO 0016+731 from observations on 6 December 1997 (1997.93) and 28 May 1999 (1999.41). Axes are relative $\alpha$ and $\delta$ in mas. See Table 1 for contour levels, beam sizes (bottom left in the maps) and peak flux densities. See Table 2 for component parametrization.

a Hubble constant $H_{0}=65 \mathrm{hms}^{-1} \mathrm{Mpc}^{-1}$ and a deceleration parameter $q_{0}=0.5$, which results in the linear scales $(10 \mathrm{pc})$ plotted on the bottom right corner of each image.

\section{1. $Q S O 0016+731$}

The QSO 0016+731 has an optical magnitude $V=19.0$ and a redshift $z=1.781$ (Stickel \& Kühr 1996). Eckart et al. (1987) reported that almost all of its flux density originates in an area smaller than 3 mas and that it does not have extended structure with flux density larger than $0.1 \mathrm{Jy}$ at $5 \mathrm{GHz}$. Pearson \& Readhead (1988) confirmed this result. This source experienced a decrease of the flux density through the nineties, from $\sim 1.5$ Jy for the period 1990-1995 to $\sim 0.4$ Jy for the second half of the decade.

Our maps (Fig. 2) show a double structure along $\mathrm{PA} \sim 130^{\circ}$ at both epochs. However, an alteration in the morphology of the source is clearly seen between the first and the second epoch. The total mapped flux density decreased from $528 \mathrm{mJy}$ to $387 \mathrm{mJy}(\sim 27 \%)$. This decrease in the total flux density of the source is due to a strong decrease in the flux of the westernmost component (XB, the brightest feature in Fig. 2). To quantify those changes, we used the tasks MODELFIT and MODFIT of the Caltech package (Pearson 1991) to fit the visibility data to a model of elliptical components with Gaussian brightness profiles. The models are presented in Table 2. The modeling provides reasonable fits with three components: a compact one (XA), a second, more elongated one (XB) west of XA, and a third, weaker one (XC), the extended emission south of XA. The changes in the maps are likely associated to component XB, whose flux density decreased from 367 mJy to 205 mJy (45\%), while the emission of component XA remained more stable.

\section{2. $Q S O 0153+744$}

The QSO 0153+744, has $V=16.0$ and $z=2.338$ (Stickel \& Kühr 1996). At kiloparsec-scales, this source does not display large structures, Hummel et al. (1997). At pcscales, QSO 0153+744 is a compact double radio source embedded in a halo, with a separation of 10 mas between components (Eckart et al. 1987; Hummel et al. 1988; Pearson \& Readhead 1988). The jet, as seen in projection, changes its direction by a full $180^{\circ}$ between component $\mathrm{A}$ and the bright secondary component $\mathrm{B}$, located about 10 mas from the core at $\mathrm{PA} \sim 150^{\circ}$. No component motion has been detected. The source flux density at 8.4 GHz has been roughly stable at $\sim 0.8 \mathrm{Jy}$, from 1996 to 1999.

Figure 3 shows the two components reported by Eckart et al. (1987) (XA and XB in the figure), and emission in an arc joining the two components. Both XA and XB are well modeled at each epoch by four Gaussian individual components (Table 2). The complex structure of this radio source does not show dramatic changes over the years, although some slight changes are readily visible. The overall flux density has been decreasing slowly in the second half of the nineties (Peng et al. 2000). A careful 
Table 1. Map parameters for sources of the complete S5 polar cap sample.

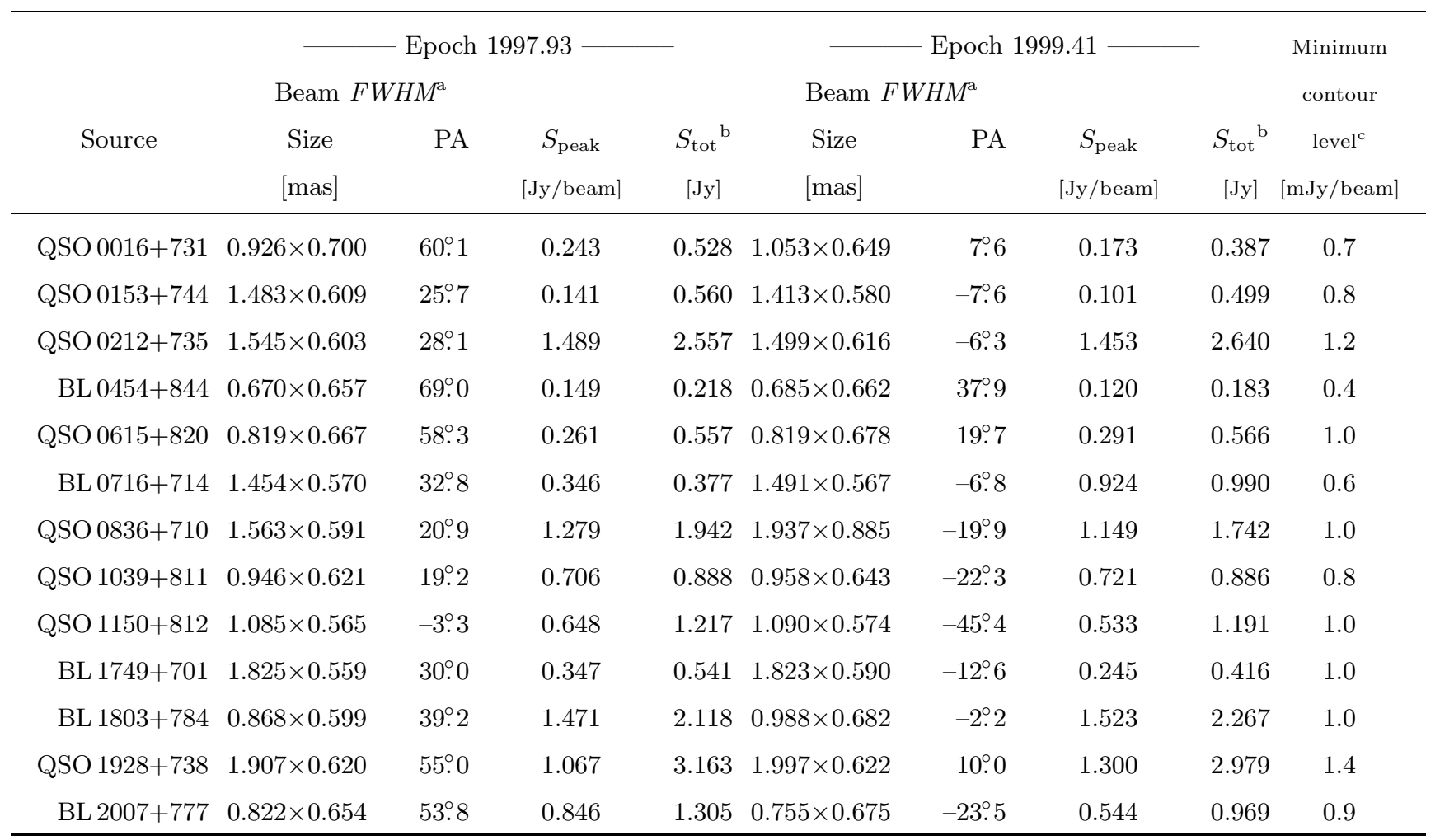

a The restoring beam is an elliptical Gaussian with full-width-half-maximum $(F W H M)$ axes $a \times b$. For each source, the position angle (PA) stands for the direction of the major axis (measured north through east).

b Total flux density recovered in the hybrid mapping process.

c Contours in the maps of the figures shown in Sects. 3.1 to 3.13 are the tabulated value times $(-1,1, \sqrt{3}, 3,3 \sqrt{3}, \cdots)$.

astrometric test of the registration of the maps (Ros et al., in preparation), should shed more light on eventual proper motions in the components of this source.

\section{3. $Q S O 0212+735$}

The QSO $0212+735$ has $V=19.0$ and $z=2.367$ (Stickel \& Kühr 1996). This source does not show extended emission at kpc scales in VLA images obtained at $1.49 \mathrm{GHz}$ (Antonucci et al. 1986). Eckart et al. (1987) reported a core-jet structure with a jet extending 12.5 mas away from the core in PA $91^{\circ}$ at $1.7 \mathrm{GHz}$. Pearson \& Readhead (1988) reported a very similar structure to that found by Eckart et al. (1987). More recently, Fey et al. (1996) reported maps at $2.3 \mathrm{GHz}$ that show an elongated jet with a strong component at 13 mas $\left(\mathrm{PA} 91^{\circ}\right)$ of the core and a fainter component at 41 mas (PA $\left.95^{\circ}\right)$ from the core. Their 8.4 GHz map shows extended emission up to 14 mas, in PA $42^{\circ}$.

Our maps at $8.4 \mathrm{GHz}$ (Fig. 4) display a jet-like structure prolonging eastwards up to 14 mas. We reproduce the emission of the radio source at each epoch with a 7-component model (Table 2). The peak of brightness corresponds to component XB. In the first epoch, XA is at $\sim 0.5$ mas northwest of $\mathrm{XB}$, in $\mathrm{PA} \sim 55^{\circ}$. In the second epoch, some structure is visible west of the brightest region $\mathrm{XA} / \mathrm{XB}$.

Lacking an accurate astrometric registration, all components seem to have remained unchanged for the last 20 yrs. Note that component XG, about 13.8 mas (PA $\sim 93^{\circ}$ ) east of the peak of brightness, with a $3 \%$ of the total mapped flux density, appears unchanged between our two epochs.

\section{4. $B L 0454+844$}

The BL Lacertae object BL $0454+844$, with $V=16.5$ and $z=0.112$ (Stickel \& Kühr 1996) has been reported to contain all the flux density in its milliarcsecond structure (Eckart et al. 1987). Those authors reported a strong core emission and weaker extended emission southwards, which was also confirmed by Pearson \& Readhead (1988). Its radio emission shows variability on scales of (several) months. It registered a local maximum in mid 1995, and 
Table 2. Elliptical Gaussian component model parameters for the radio sources of the complete S5 polar cap sample.

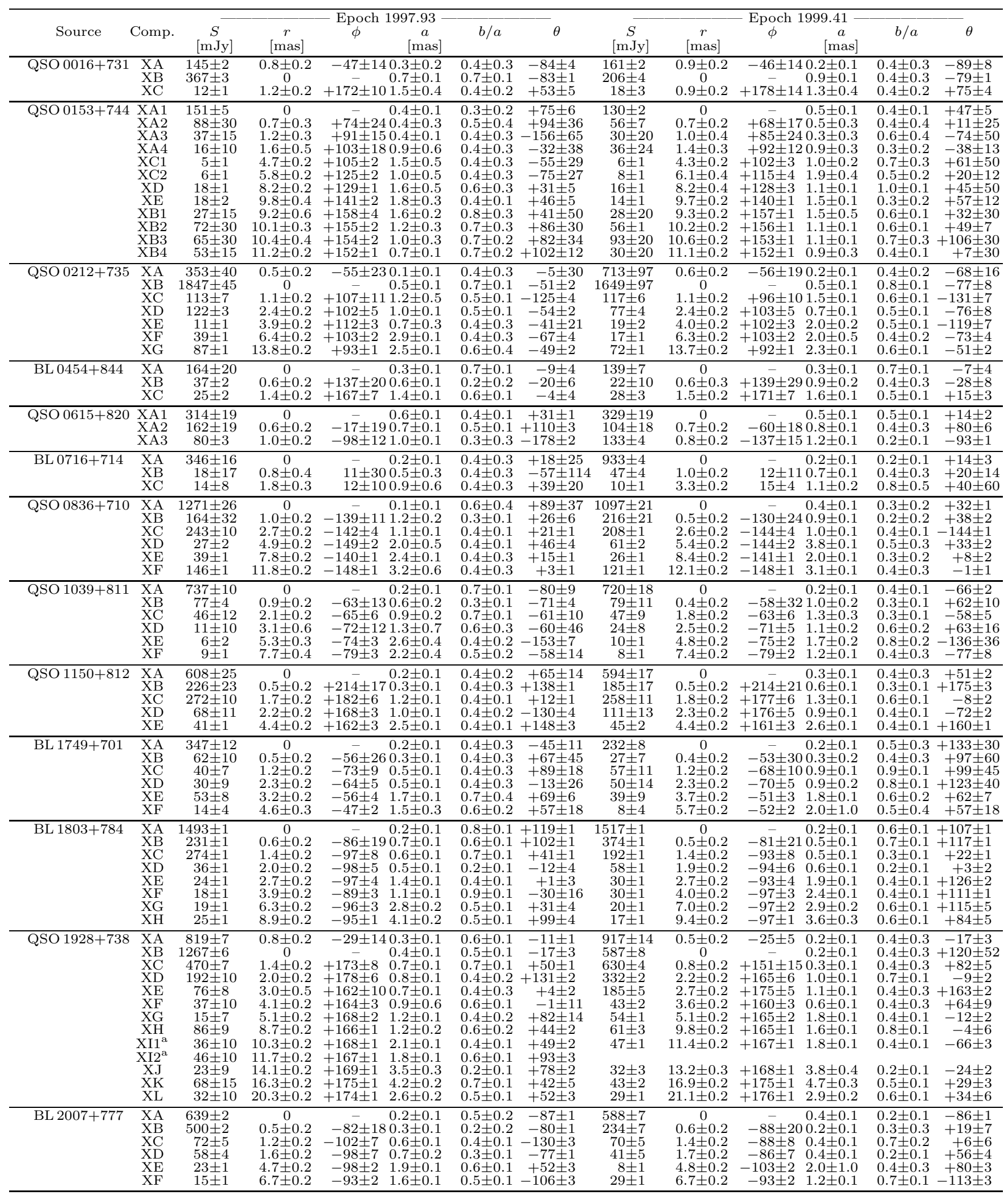

Note: For each source whose name is on the first column, the other columns correspond to the component designation (as indicated in Figs. 2 to 16), flux density, separation and position angle relative to the strongest component, major axis, axis ratio, and position angle of the major axis. The given uncertainties have been estimated from the exploration of the parameter space $(S, r, \Phi, a, b / a$, and $\theta)$, and from empirical considerations (e.g., the errors of the component positions are typically smaller than a tenth of a beam width, but to be conservative we have assumed a magnitude of a fifth of beam width for them).

a This component is model fitted as double only in the first epoch. In the second epoch the model fit algorithm merged both components into a single one. 

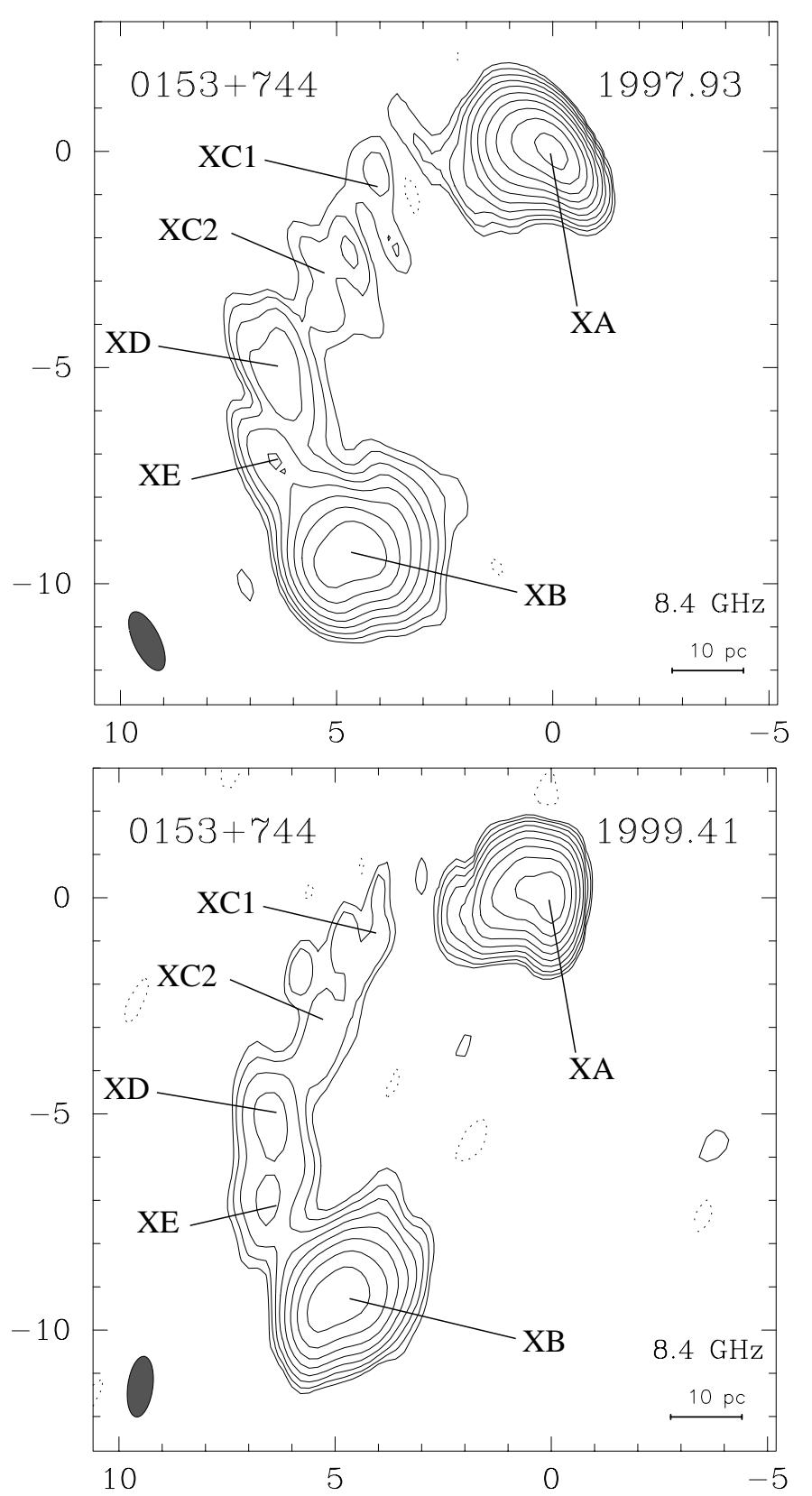

Fig. 3. VLBA images of QSO $0153+744$ from observations on 6 December 1997 (1997.93) and 28 May 1999 (1999.41). Axes are relative $\alpha$ and $\delta$ in mas. See Table 1 for contour levels, synthesized beam size (bottom left in the figure), and peak flux densities. See Table 2 for component parametrization.

it has since then been decreasing (by $\sim 40 \%$ ) through late 1999. Then, its flux density started to increase again (Peng et al. 2000). Our maps (Fig. 5) show a very compact structure. There is no emission outside $\sim 4$ mas of the core. We reproduce its emission with a three-component model (see Table 2). It is interesting to notice that the most compact source in the sample has also the smallest redshift.
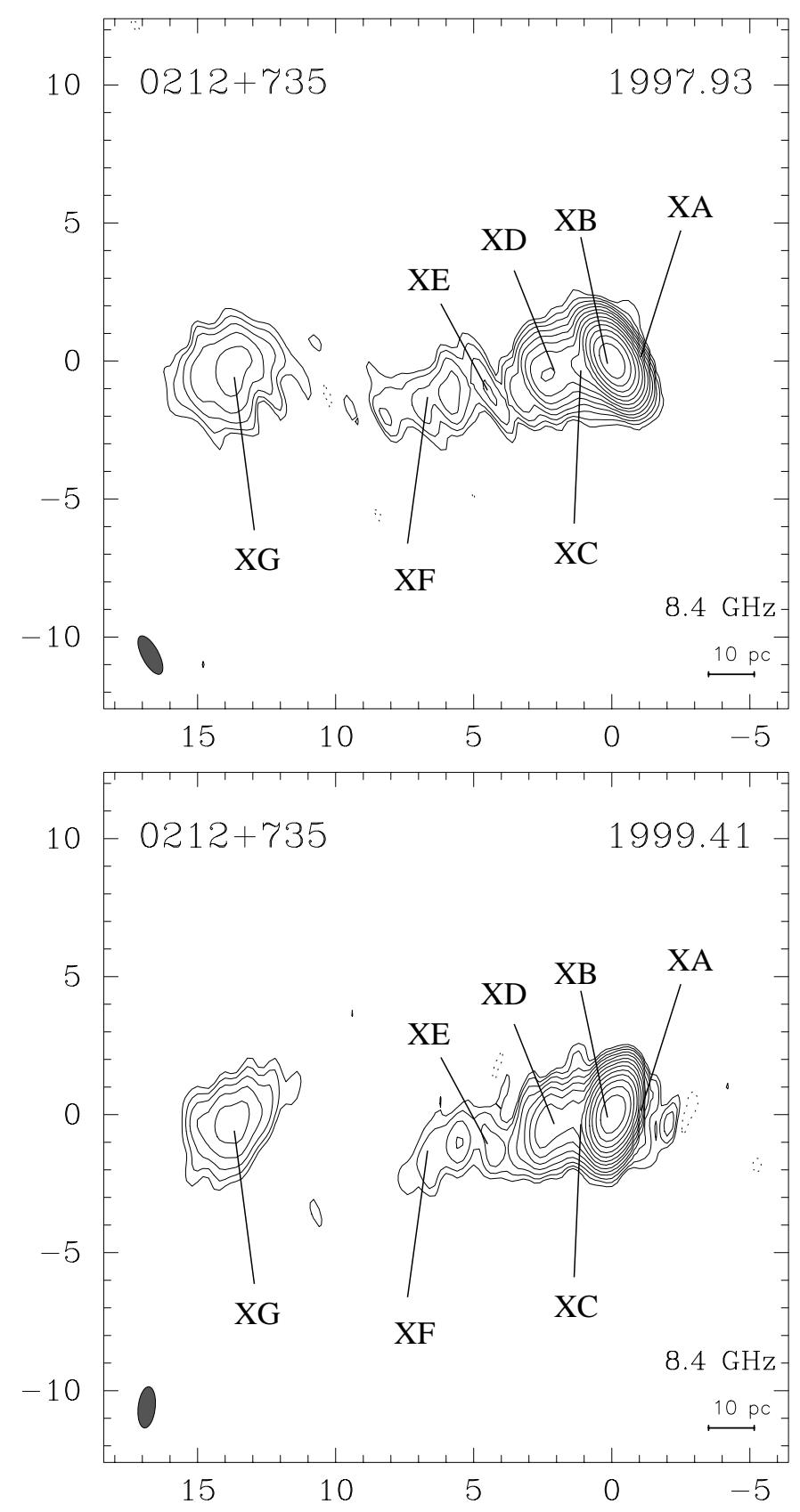

Fig. 4. VLBA images of QSO $0212+735$, observed on 6 December 1997 (1997.93) and 28 May 1999 (1999.41). Axes are relative $\alpha$ and $\delta$ in mas. See Table 1 for contour levels, synthesized beam size (bottom left in the figure), and peak flux densities. See Table 2 for component parametrization.

\section{5. $Q S O 0615+820$}

The QSO $0615+820$ is a radio source with $V=17.5$ and $z=0.710$ (Stickel \& Kühr 1996). Eckart et al. (1987) reported this radio source to be unresolved, and modeled it with a single elliptical Gaussian profile elongated in $\mathrm{PA} 195^{\circ}$ at $5 \mathrm{GHz}$ and in $\mathrm{PA} 181^{\circ}$ at $15 \mathrm{GHz}$. 

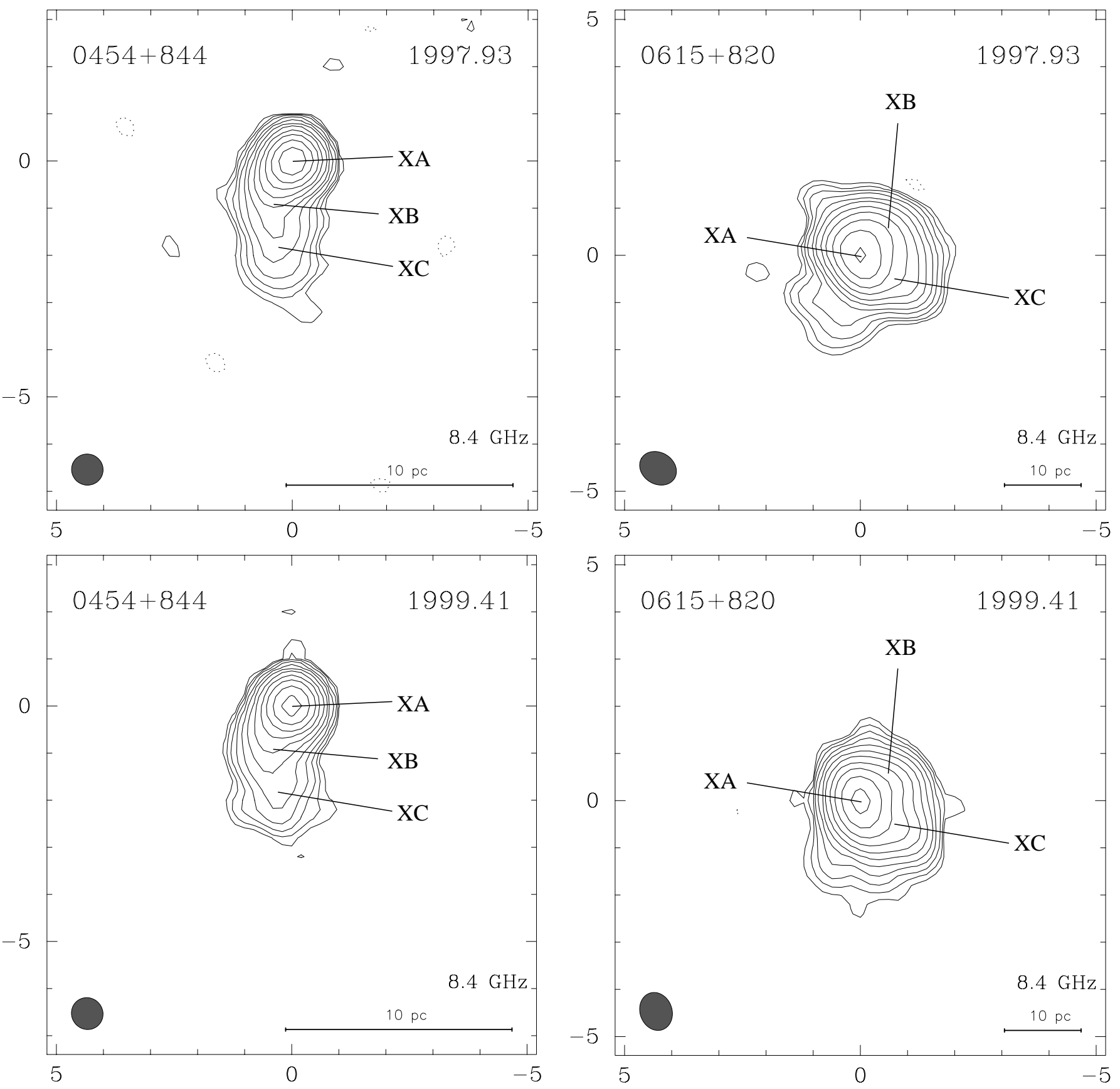

Fig. 5. VLBA images of BL $0454+844$, observed on 6 December 1997 (1997.93) and 28 May 1999 (1999.41). Axes are relative $\alpha$ and $\delta$ in mas. See Table 1 for contour levels, synthesized beam sizes (bottom left in the maps), and peak flux densities. See Table 2 for component parametrization.

Fig. 6. VLBA images of QSO $0615+820$, observed on 6 December 1997 (1997.93) and 28 May 1999 (1999.41). Axes are relative $\alpha$ and $\delta$ in mas. See Table 1 for contour levels, synthesized beam sizes (bottom left in the maps), and peak flux densities. See Table 2 for component parametrization.

Our maps (Fig. 6) show a compact plateau-like structure within 2-3 mas; however, we could not reasonably fit the brightness distribution with a single elliptical Gaussian component. We obtained good Gaussian fits (Table 2) with three close components within 1 mas,

with a spatial L-shaped distribution, being the brightest one XA, to the east. 


\section{6. $B L 0716+714$}

The object BL $0716+714(V=14.17)$ is a rapidly variable source across the electromagnetic spectrum, a paradigm of the intra-day variable (IDV) behavior (Quirrenbach et al. 1991; Wagner et al. 1996). No sign of optical counterpart exists, and thus no redshift is available. This fact prompted several authors (e.g., Eckart et al. 1987) to assume a value of $z>0.3$. Previous radio maps show a corehalo structure on arcsecond scales (Antonucci et al. 1986). VLBI maps at 5 and $22 \mathrm{GHz}$ show a very compact source on the milliarcsecond-scale with some hints of a core-jet structure oriented along a $\mathrm{PA} \sim 10^{\circ}$ (Eckart et al. 1987).

Our maps (Fig. 7) show a core-jet structure extended northwards. We modeled the structure with three components (see Table 2). In the first epoch the components lie at 0.8 mas $(\mathrm{XB})$ and 1.8 mas $(\mathrm{XC})\left(\mathrm{PA} \sim 11^{\circ}\right)$ of the main component (XA), and in the second epoch, at 1.0 and 3.3 mas (again $\mathrm{PA} \sim 11^{\circ}$ ). Taking the association at face value, and for the elapsed period of time between both epochs $(\sim 1.5 \mathrm{yr}), \mathrm{XC}$ would have moved with a rate

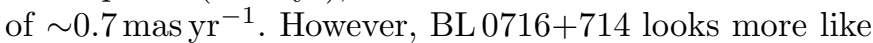
a source with "flaming" features than the "classical" corejet structure with components emerging from the core and traveling along the jet. In a classical core-jet source the emergence of components is related to changes in the flux density. For this source, the flux density has changed dramatically from one epoch to the other (University of Michigan Radio Astronomy Observatory data base and Peng et al. 2000). After smooth flux density changes from 1994 to 1997, the flux density began to rise by mid 1997 . A decrease followed by late 1997, and a rise of more than $100 \%$ by the first half of 1998 , remaining stable over the following months until a further decrease took place by mid 1999. We mapped a total flux density of $0.377 \mathrm{Jy}$ in 1997.93, and 0.990 Jy in 1999.41. This source is a case where frequent sampling of the structure and astrometric registration are essential to correctly interpret the source structure changes.

\section{7. $Q S O 0836+710(4 C 71.07)$}

The QSO 0836+710 has $V=16.5$ and $z=2.172$ (Stickel \& Kühr 1996). At kpc-scales, it displays a highly polarized secondary component 1.3 arcsec off the core at $\mathrm{PA} \sim 200^{\circ}$ (Perley 1980; O'Dea et al. 1988). MERLINVLBI images at 90 and $18 \mathrm{~cm}$ show a jet structure extending up to 150 mas in direction to the outer arcsecond lobe (Hummel et al. 1992b). It shows a complex and wiggled one-sided core-jet structure along $\mathrm{PA} \sim 215^{\circ}$ at pc-scales. The structure of the jet can be characterized by a sequence of kinks among which the jet follows a slightly curved path (Krichbaum et al. 1990). Based on data at 8.4, 15, 22, and $86 \mathrm{GHz}$, Otterbein et al. (1998) reported the ejection of a new component at mas scales around
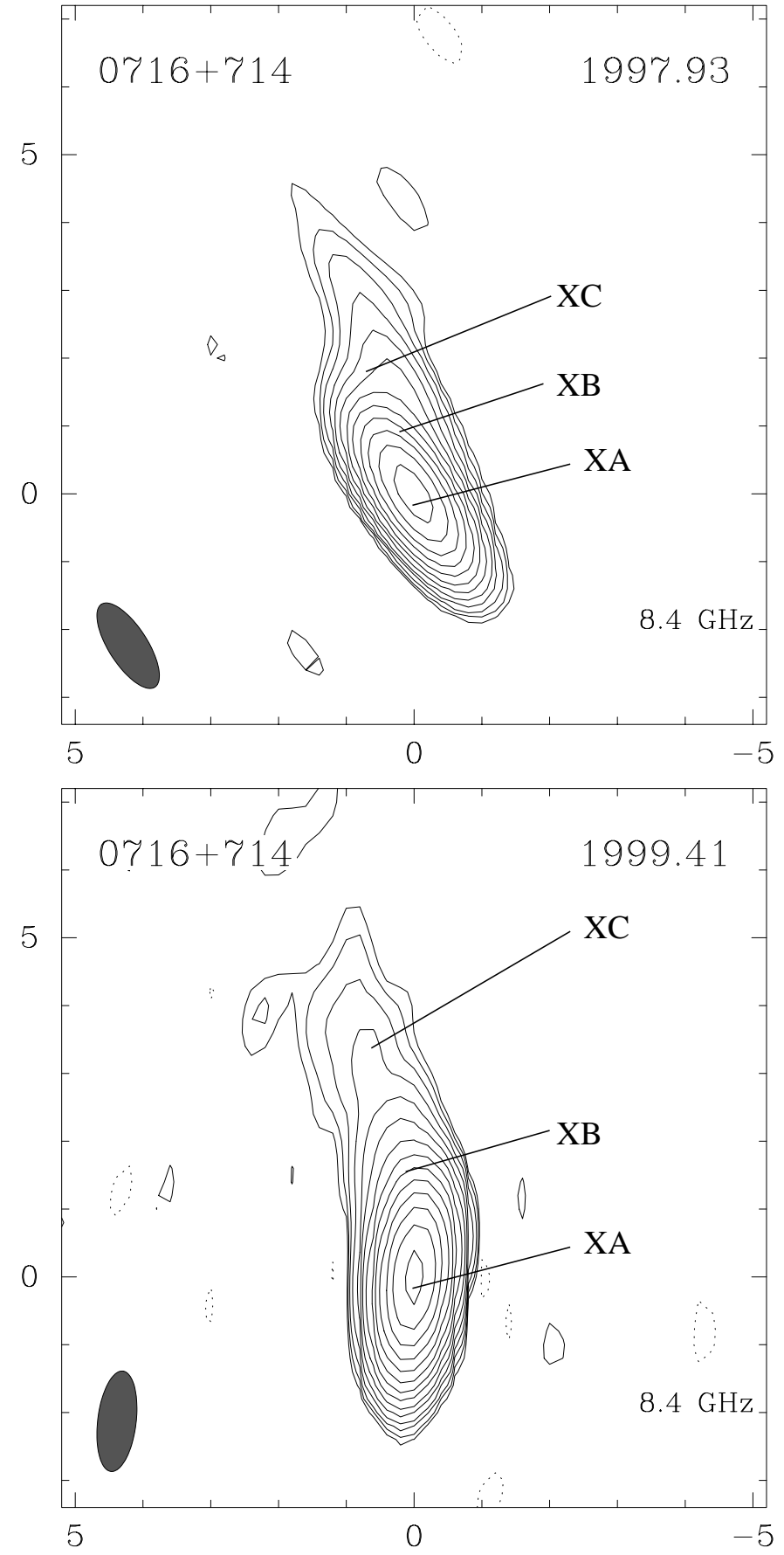

Fig. 7. VLBA images of BL $0716+714$, observed on 6 December 1997 (1997.93) and 28 May 1999 (1999.41). Axes are relative $\alpha$ and $\delta$ in mas. See Table 1 for contour levels, synthesized beam sizes (bottom left in the maps), and peak flux densities. See Table 2 for component parametrization.

epoch 1992.65, with an apparent superluminal motion of $\mu=0.26 \pm 0.03$ mas $y r^{-1}$. The ejection of this component would be directly related to gamma-, X-ray, and optical activity observed in February 1992 (Otterbein et al. 1998). 
Although our maps (Fig. 8) are convolved with differently oriented beams, the features in the maps can be identified unambiguously. We detect emission up to 30 mas away of the core, although we plot the structure only extending 15 mas from the core to see more clearly the main features. We reproduce the emission up to $\sim 12$ mas from the core with six components. We convolved our images with a circular beam of 0.6 mas size to better compare the inner structure changes, and show them in Fig. 9. We can neither confirm nor reject the superluminal motion reported by Otterbein et al. (1998), until an astrometric registration between our both images becomes available. Only from our model fitting it is difficult to establish proper motions between components (Table 2).

\subsection{QSO $1039+811$}

The QSO $1039+811$ has $V=16.5$ and $z=1.264$ (Stickel \& Kühr 1996). Eckart et al. (1987) reported a pc-scale core-jet structure with a jet oriented with $\mathrm{PA} \sim-70^{\circ}$ at $5 \mathrm{GHz}$. This radio source displays a misalignment between the pc-scale and the kpc-scale structure (Appl et al. 1996).

Our maps (Fig. 10) show extended jet structure up to 10 mas away of the core component. The multi-frequency flux density monitoring reported by Peng et al. (2000) shows a rise from 1997 to 1999. Our maps, however, have almost the same flux density for both epochs (0.888 and $0.886 \mathrm{Jy}$ at 1997.93 and 1999.41 , respectively). The model fitting (Table 2) reports an apparent backwards motion of the components XB (from 0.9 to 0.4 mas from XA), XC (2.1 to 1.8 mas), XD (3.1 to 2.5 mas), XE (5.3 to 4.8 mas), and XF (7.7 to 7.4 mas). This apparently contracting motion could be related to changes in the core region. The emergence of a component, in its early stage, can produce apparent backward motions of the rest of the jet components at cm-wavelengths (see, e.g., Guirado et al. 1998; Ros et al. 1999 for QSO 1928+738). Those effects should be tested after the astrometric alignment of the images becomes available. Note that the moving components are very weak in comparison with the main feature (which contains more than the $80 \%$ of the mapped flux density in both cases), and also that the $(u, v)$-sampling, and consequently the synthesized beams, differ substantially from the first epoch to the second.

\section{9. $Q S O 1150+812$}

The QSO $1150+812$ has $V=18.5$ and $z=1.250$ (Stickel $\&$ Kühr 1996). Its radio structure has been studied at pc and kpc scales by Appl et al. (1996), who reported a misalignment of the jet between both scales. At kpc scales, Murphy et al. (1993) reported a VLA map with a core and a fainter component at 6 arcsec and $\mathrm{PA} \sim-100^{\circ}$. At pc scales, Eckart et al. (1987) reported a core-jet structure in the VLBI maps, with a faint jet emission oriented at

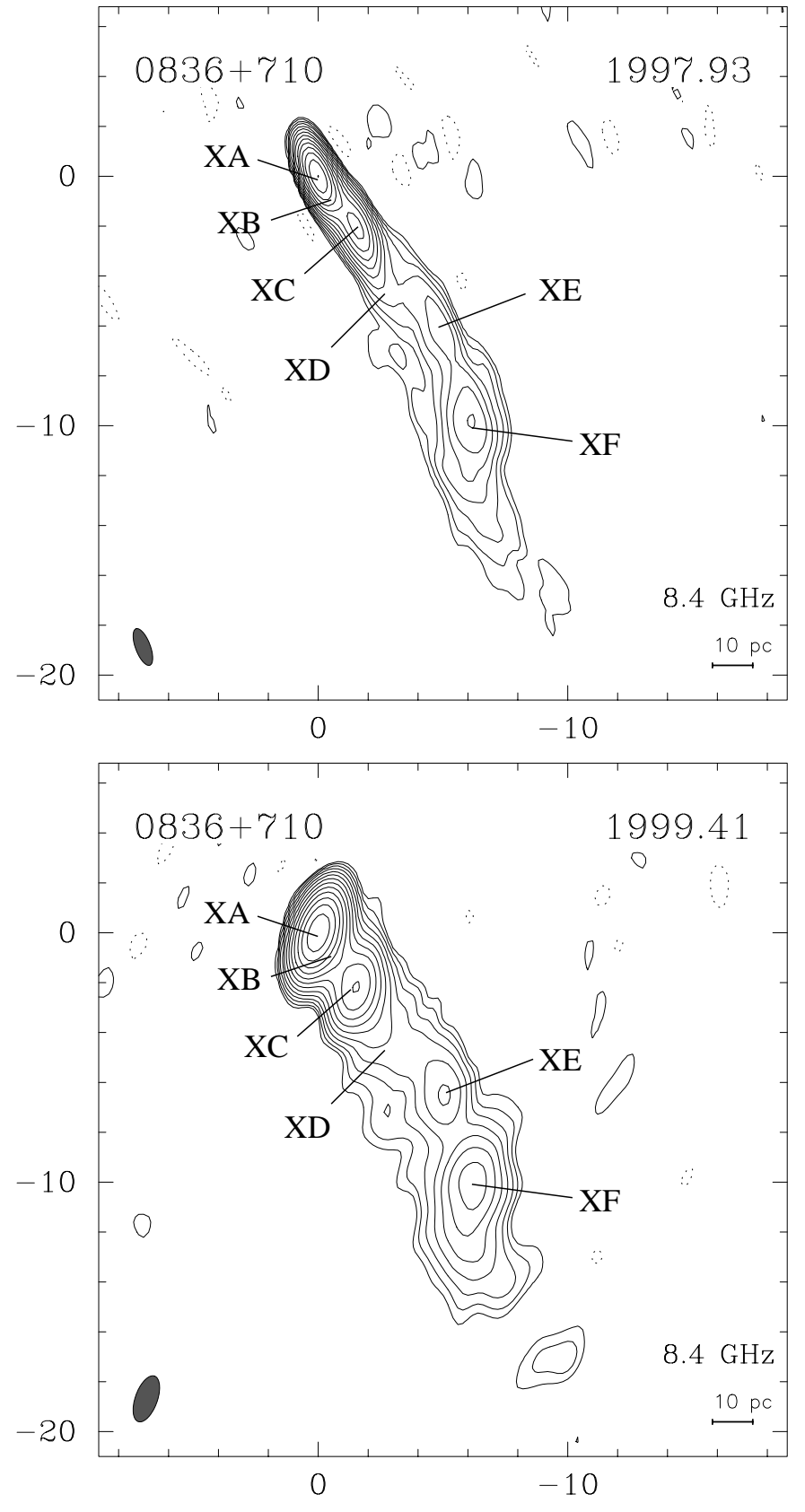

Fig. 8. VLBA images of QSO $0836+710$, observed on 6 December 1997 (1997.93) and 28 May 1999 (1999.41). Axes are relative $\alpha$ and $\delta$ in mas. See Table 1 for contour levels, synthesized beam sizes (bottom left in the maps), and peak flux densities. See Table 2 for component parametrization.

$\mathrm{PA} \sim 178^{\circ}$, and superluminal motion of about $0.12 \mathrm{mas} \mathrm{yr}^{-1}$ of the jet component between 1979.93 and 1983.25 epochs. Fey \& Charlot (1997) published VLBI maps from epoch 1995.20 epoch at 8.4 and $2.3 \mathrm{GHz}$. Extended emission is detected up to 30 mas $\left(\mathrm{PA}\right.$ of $\left.135^{\circ}\right)$ in their $2.3 \mathrm{GHz}$ maps. In their $8.4 \mathrm{GHz}$ maps, the emission extends $\sim 7$ mas $\left(\mathrm{PA} \sim 135^{\circ}\right)$. 


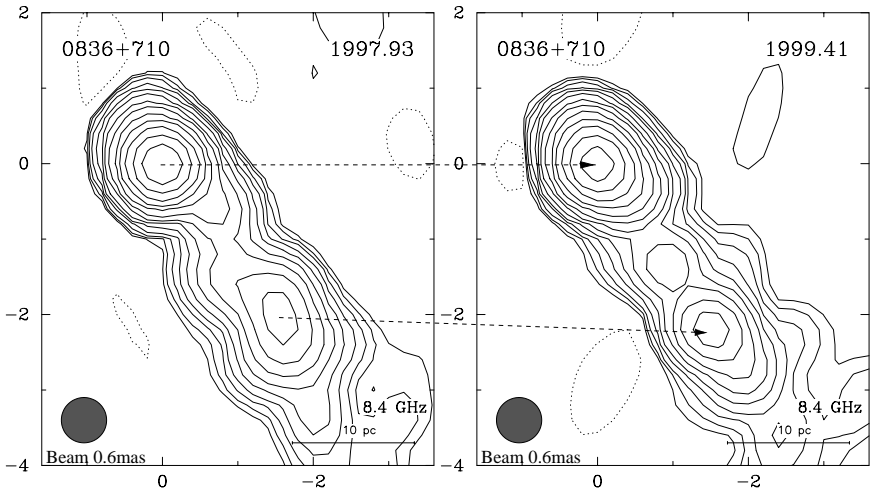

Fig. 9. VLBA images of QSO $0836+710$, convolved with a 0.6 mas circular beam. The map parameters are the same as in Fig. 8. Note the small structural changes in the inner part of the jet. The peak of brightness changes from $1.233 \mathrm{Jy} /$ beam at 1997.93 to $1.050 \mathrm{Jy} /$ beam at 1999.41 . The dashed lines draw a tentative association between features from one epoch to another.

Pérez-Torres et al. (2000) studied this radio source astrometrically referenced to BL $1803+784$, and thus our observations constitute a second and third astrometric epochs for the pair QSO 1150+812/BL 1803+784. A comparison of the astrometric results will be published elsewhere.

Our maps (Fig. 11) display a jet oriented southwards, up to 5 mas away from the core component. We model fit the source (Table 2), with 5 components in both cases, up to 4.4 mas south of the radio brightest component XA $\left(\mathrm{PA} \sim 160^{\circ}\right)$. The source increased its total flux density by less than a $20 \%$ from one epoch to the other (Peng et al. 2000), and the VLBA flux densities in our images are very similar. The radio source does not present important structural changes between our epochs, and the modelfitted components do not permit reporting of any significant proper motion.

\subsection{0. $B L 1749+701$}

The object BL $1749+701$ has $V=16.5$ and $z=0.770$ (Stickel \& Kühr 1996). VLA images at $5 \mathrm{GHz}$ (Perley 1982) show a halo of $\sim 0.4$ arcsec. O'Dea et al. (1988) reported two components at pc-scales at $15 \mathrm{GHz}$ separated by $0 .^{\prime \prime} 4$ at a PA $-150^{\circ}$. Kollgaard et al. (1992) did not report extended emission, although they found evidence of emission to the north. VLBI observations from Eckart et al. (1987) show the core, and a component at $\sim 2.4$ mas and $\mathrm{PA}-45^{\circ}$. Witzel et al. (1988) reported

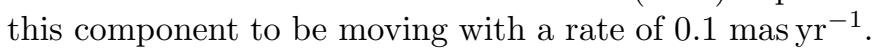
Bååth \& Zhang (1992) reported a more complicated jet structure, with a position angle of $\sim-65^{\circ}$, and gave a three-component model for maps from 1980 to 1983 . They

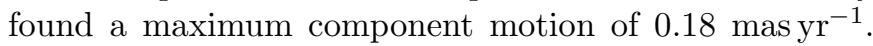
Gabuzda et al. (1992) reported new results from epoch
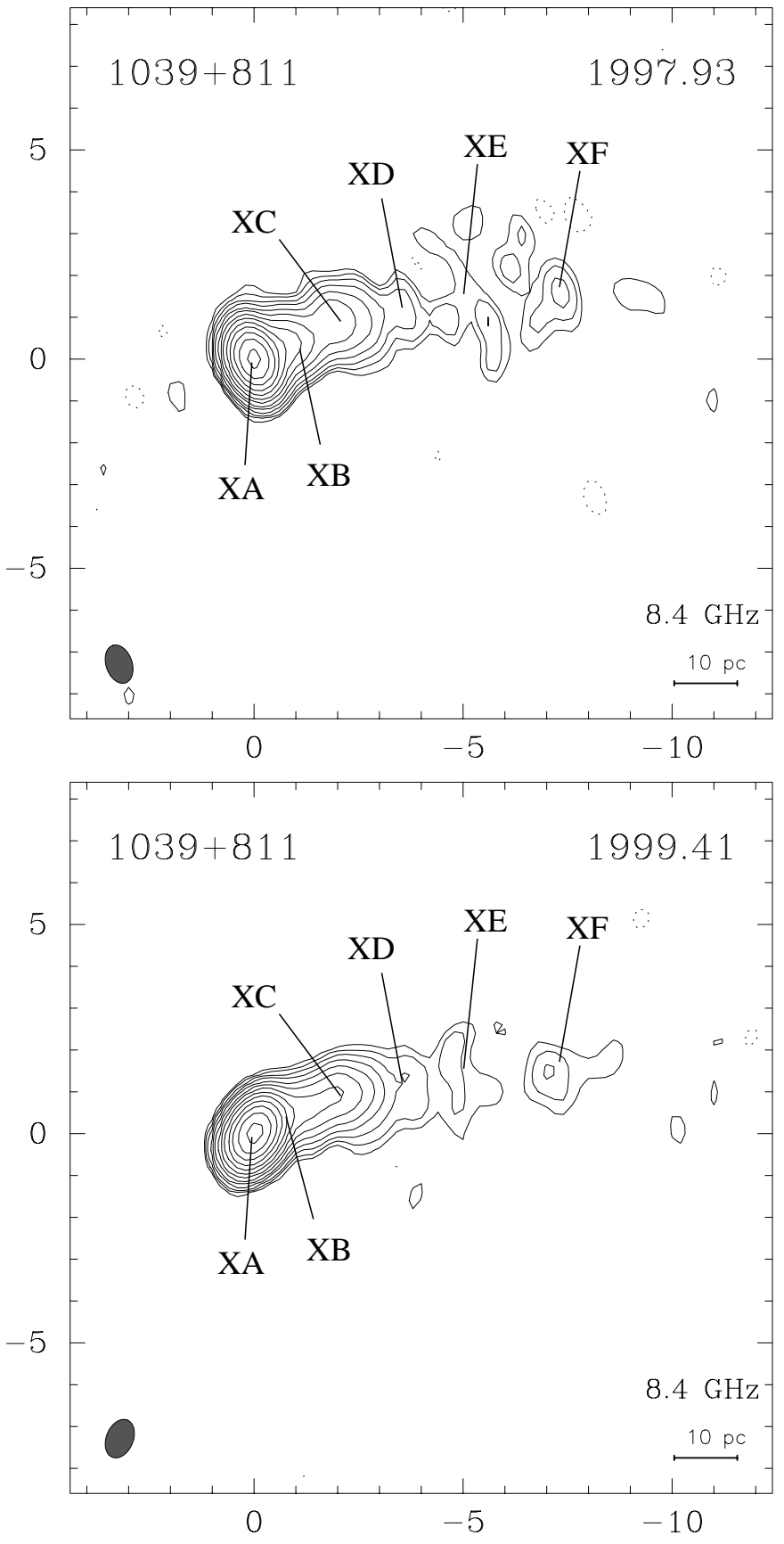

Fig. 10. VLBA images of QSO $1039+811$, observed on 6 December 1997 (1997.93) and 28 May 1999 (1999.41). Axes are relative $\alpha$ and $\delta$ in mas. See Table 1 for contour levels, synthesized beam sizes (bottom left in the figure), and peak flux densities. See Table 2 for component parametrization.

1987, and gave a 4-component model, at separations of $0.84,1.93$, and 3.41 mas of the core component (PA of $-60.7^{\circ},-66.5^{\circ}$, and $-53.0^{\circ}$, respectively). These authors claimed that the radio source has an unusually low degree of polarization for BL Lacertae objects, and also questioned some of the proper motions reported above. 

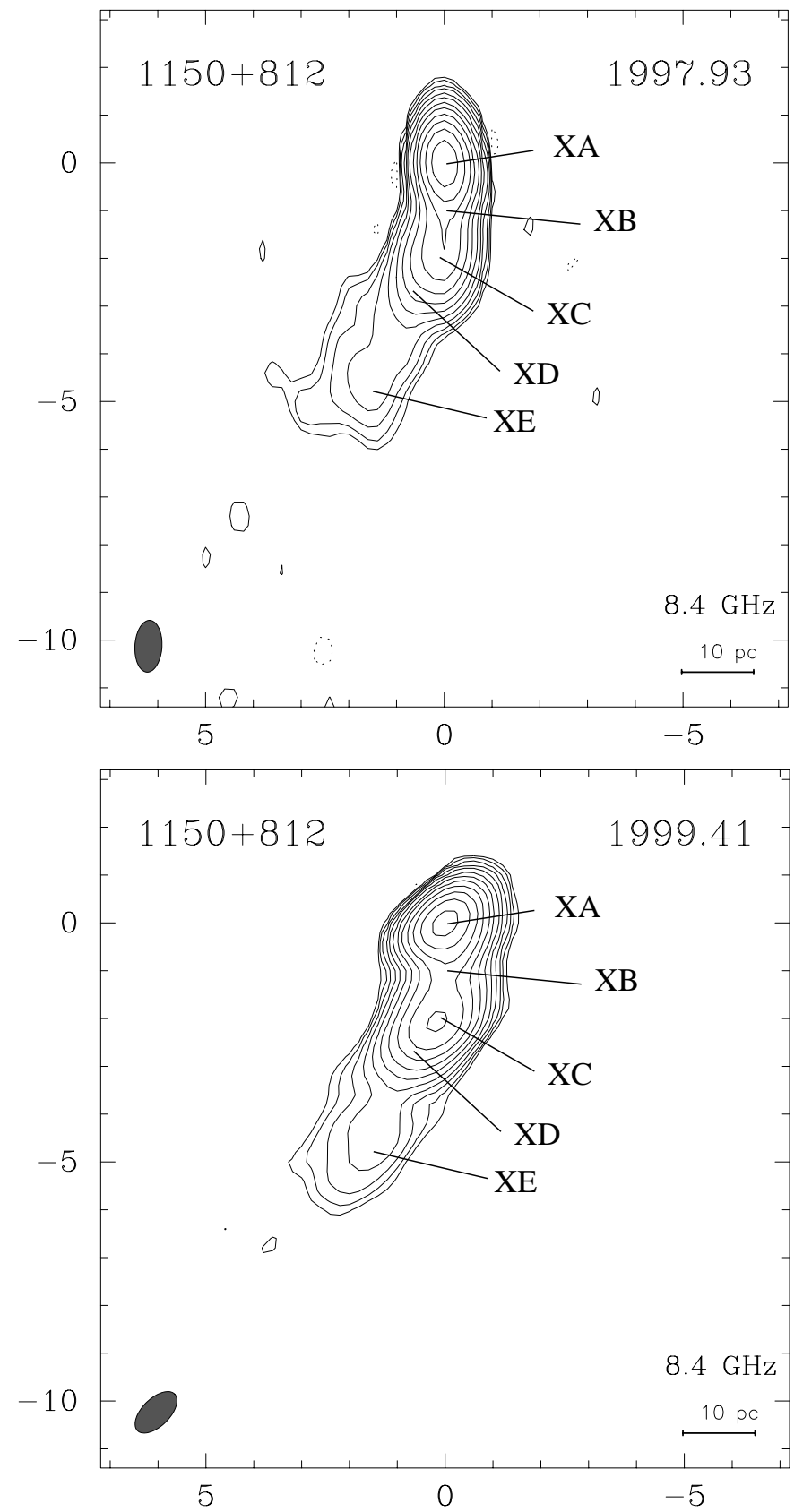

Fig. 11. VLBA images of QSO $1150+812$, observed on 6 December 1997 (1997.93) and 28 May 1999 (1999.41). Axes are relative $\alpha$ and $\delta$ in mas. See Table 1 for contour levels, synthesized beam sizes (bottom left in the maps), and peak flux densities. See Table 2 for component parametrization.

Our maps (Fig. 12) show a complex jet structure oriented to the northwest, and extended up to 7 mas from the core. We identify in our images more components than the number reported by (Bååth \& Zhang 1992). We observe about the same structure for the two epochs, the beam being perpendicular to the jet direction in the first epoch, and (unfortunately) more aligned to it for the second one. A Gaussian model of the visibilities (see Table 2) includes a compact, central component (XA), and 5 components, XB to XF, stretching up $\sim 5$ mas from XA, and describing a slightly curved trajectory northwards. This source offers interesting prospects for study: the astrometric analysis will unambiguously prove the proper motions early reported for this source and will also provide insight into the nature of the XA component.

\subsection{1. $B L 1803+784$}

The object BL $1803+784$ has $V=16.4$ and $z=0.864$ (Stickel \& Kühr 1996). It has been extensively studied in the past at different radio wavelengths. At kpc scales, the maps reveal components south of the core at 2, 37, and 45 arcsec (Antonucci et al. 1986; Strom \& Biermann 1991; Murphy et al. 1993). Eckart et al. (1987) and Pearson \& Readhead (1988) reported a core-jet structure at pc scales with a jet oriented westwards. Schalinski (1990) suggests the stationarity of a component at 1.2 mas of the core. Krichbaum et al. $(1993,1994)$ with $43 \mathrm{GHz}$ observations reported the existence of some traveling components between the core and such component, now estimated to be at 1.4 mas separation. Steffen (1994) modeled the radio source as an homogeneous plasma jet of helical trajectory in adiabatic expansion. Fey et al. (1996) reported VLBA results at 8.5 and $2.3 \mathrm{GHz}$. At $8.5 \mathrm{GHz}$, the radio source displays a 12 mas jet. At $2.3 \mathrm{GHz}$, the radio source shows a jet up to 40 mas with $\mathrm{PA} \sim-110^{\circ}$. At this distance, the jet turns northwards, reaching the extended emission a PA up to $-60^{\circ}$. This radio source has been studied astrometrically by Ros et al. (1999) with reference to QSO 1928+738/BL 2007+777, and by PérezTorres et al. (2000) with reference to QSO 1150+812.

Our maps (Fig. 13) show similar features to those obtained by Fey et al. (1996). The component at 1.2-1.4 mas of the strong core is present, as it has been for all VLBI observations during the last 20 years. A model fitting of the visibilities with 8 elliptical Gaussian components (Table 2) reproduces the structure of the radio source. The inner region has three main components: XA (with $1.5 \mathrm{Jy}$, the "core"), XB (at $0.5-0.6$ mas with PA of $\sim-85^{\circ}$ ), corresponding to the component reported by Krichbaum et al. 1993), and XC (1.2 mas, $\left.\mathrm{PA} \sim-95^{\circ}\right)$. Those three components represent $\sim 95 \%$ of the flux density of the source. Further components range from $\sim 2$ to $\sim 9$ mas from XA. The jet bends to the south at $\sim 5$ mas of the main feature. At lower frequencies, the source has more extended emission at $\mathrm{PA} \sim-100^{\circ}$. Such extended emission is also present in our maps, though not shown in Fig. 13. We find no evidence for bona fide proper motions of the components from our two observing epochs. 

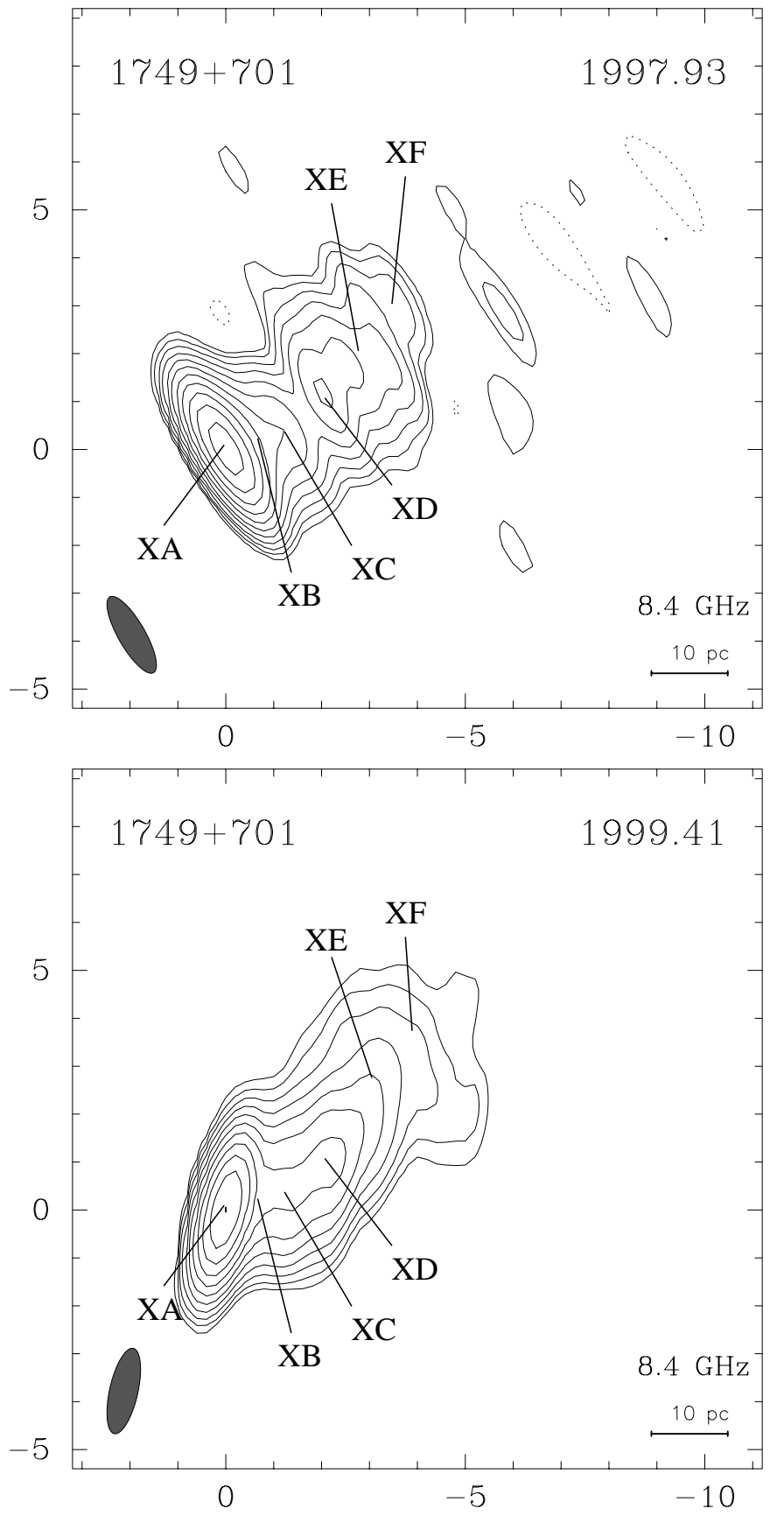

Fig. 12. VLBA images of BL $1749+701$, observed on 6 December 1997 (1997.93) and 28 May 1999 (1999.41). Axes are relative $\alpha$ and $\delta$ in mas. See Table 1 for contour levels, synthesized beam size (bottom left in the maps), and peak flux densities. See Table 2 for component parametrization.

\subsection{QSO $1928+738(4 C 73.18)$}

QSO $1928+738$ has $V=15.5$ and $z=0.3021$ (Roos et al. 1993), and displays superluminal motion in a jet oriented westwards. It is the best known and most extensively studied radio source of the complete S5 polar cap sample. At kpc scales, it displays extended emission in the
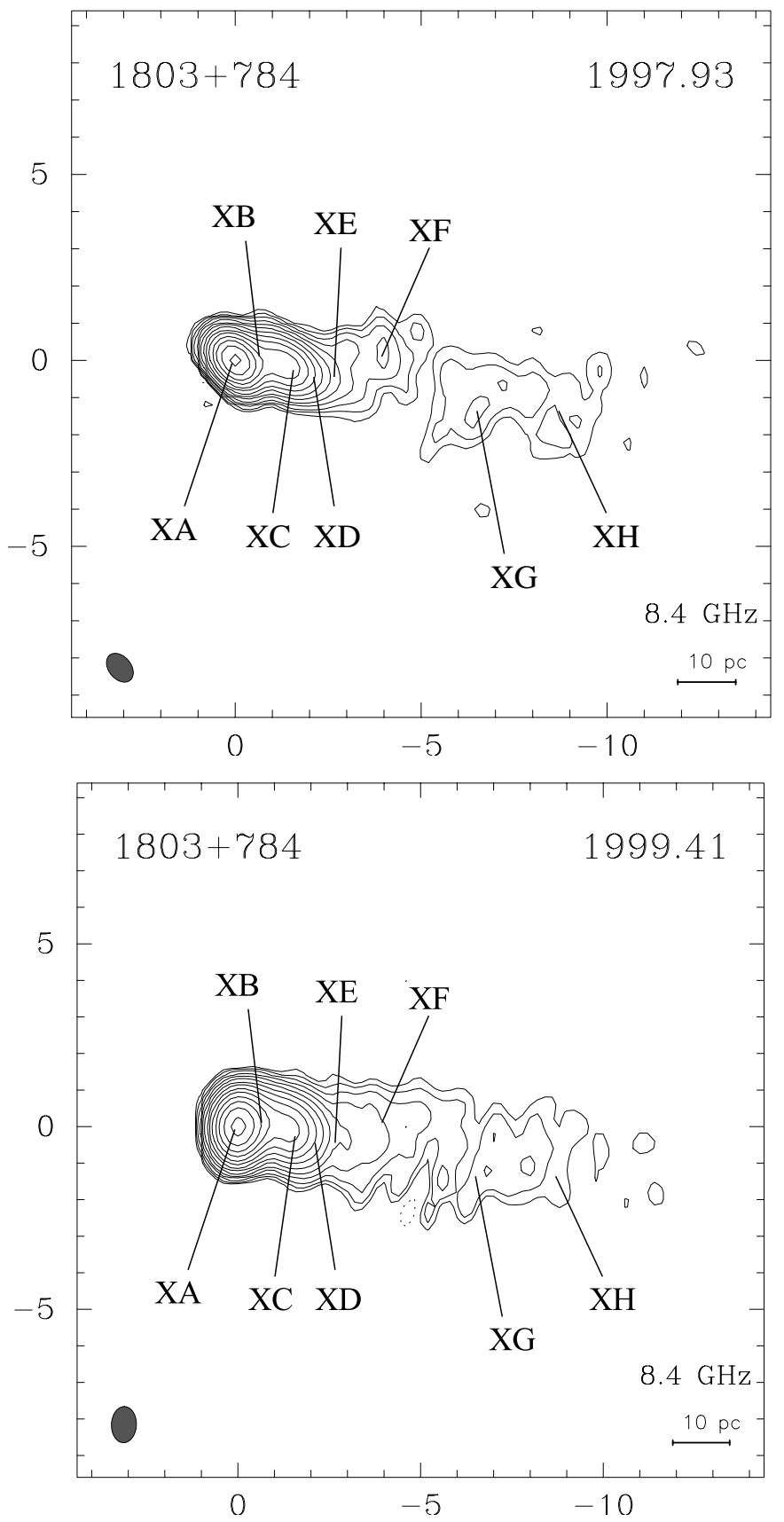

Fig. 13. VLBA images of BL $1803+784$, observed on 6 December 1997 (1997.93) and 28 May 1999 (1999.41). Axes are relative $\alpha$ and $\delta$ in mas. Contours are $0.859 \mathrm{mJy} /$ beam $\times$ $(1,2,4, \ldots, 1024)$. See Table 1 for contour levels, synthesized beam sizes (bottom left in the maps), and peak flux densities. See Table 2 for component parametrization.

north-south direction, with two lobes separated by 40 arcsec $\left(\mathrm{PA} \sim 0^{\circ}\right)$ (Rusk \& Rusk 1986). Johnston et al. (1987) and Murphy et al. (1993) detected emission up to 80 arcsec from the core. Based on kpc-scale images, Hummel et al. (1992a) modeled the radio source as an object with a 
helical magnetic field attached to a rotating accretion disk. Eckart et al. (1985) reported a self-absorbed core and a pc-scale jet 17 mas long, whose components display superluminal motion. Roos et al. (1993) modeled the radio source as a binary black hole. Guirado et al. (1995, 1998) detected a shift in the peak of brightness between different epochs, based on an astrometric analysis. Ros et al. (1999) confirmed the results of Guirado et al., and also studied the proper motions of QSO $1928+738$ by aligning astrometrically maps of 1985 and 1991.

Our maps (Fig. 14) show very elliptical synthesized beams, which nevertheless allow one to distinguish perfectly different features of its pc-scale. The flux density of QSO $1928+738$ at $8.4 \mathrm{GHz}$ has not changed much from the first to the second epoch. Hummel et al. (1992a) reported that the core of this radio source ejects a new component every $\sim 1.6$ yr (approximately the time elapsed between our two observing epochs). If we convolve the CLEANed components with a 0.6 mas circular beam (Fig. 15), structural changes are evident between the two epochs. The component XA (north of the brightest one in 1997.93), has $\sim 0.8 \mathrm{Jy}$, and XB (the brightest in 1997.93), has $\sim 1.3 \mathrm{Jy} . \mathrm{In}$ 1999.41 they have $\sim 0.9$ and $\sim 0.6$ Jy, respectively. Previous astrometric results (Guirado et al. 1998; Ros et al. 1999) show that most likely none of those components corresponds to the true "core", which should be northwards of them (shown as a question mark in Fig. 15) and whose radiation is probably very self-absorbed. If we take as reference component XB (Table 2), the rest of the components extend southwards in $\mathrm{PA} 165^{\circ}$ to 4-8 mas distance and in $175^{\circ}$ to $15-20$ mas. Though we could try to identify the components seen in 1991.88 by Ros et al. (1999) with those reported for our first epoch ( $\sim 7.1$ years later), such an attempt would be, at least, adventurous. Indeed, for an emergence rate of one component every 1.6 years, QSO $1928+738$ should have produced 4 or 5 components, making completely ambiguous any component identification. The comparison of these components with results from previous epochs by us and by other authors will be presented elsewhere.

\subsection{3. $B L 2007+777$}

The object BL $2007+777$ has $V=16.5$ and $z=0.342$ (Stickel \& Kühr 1996). This radio source has been studied with different techniques and at different frequencies in the last 20 years. Its kpc scale has been studied, e.g., by Antonucci et al. (1986), Kollgaard et al. (1992), and Murphy et al. (1993). Those authors presented maps with compact emission, and extended components at 8.5 and 15.8 arcsec to the west, and another extended component at 11 arcsec to the east, of the brightest compact component. At pc scales, the radio source is also bright. All VLBI maps (Eckart et al. 1987; Pearson \& Readhead 1988; Guirado et al. 1995; Xu et al. 1993; Fey \& Charlot 1997;
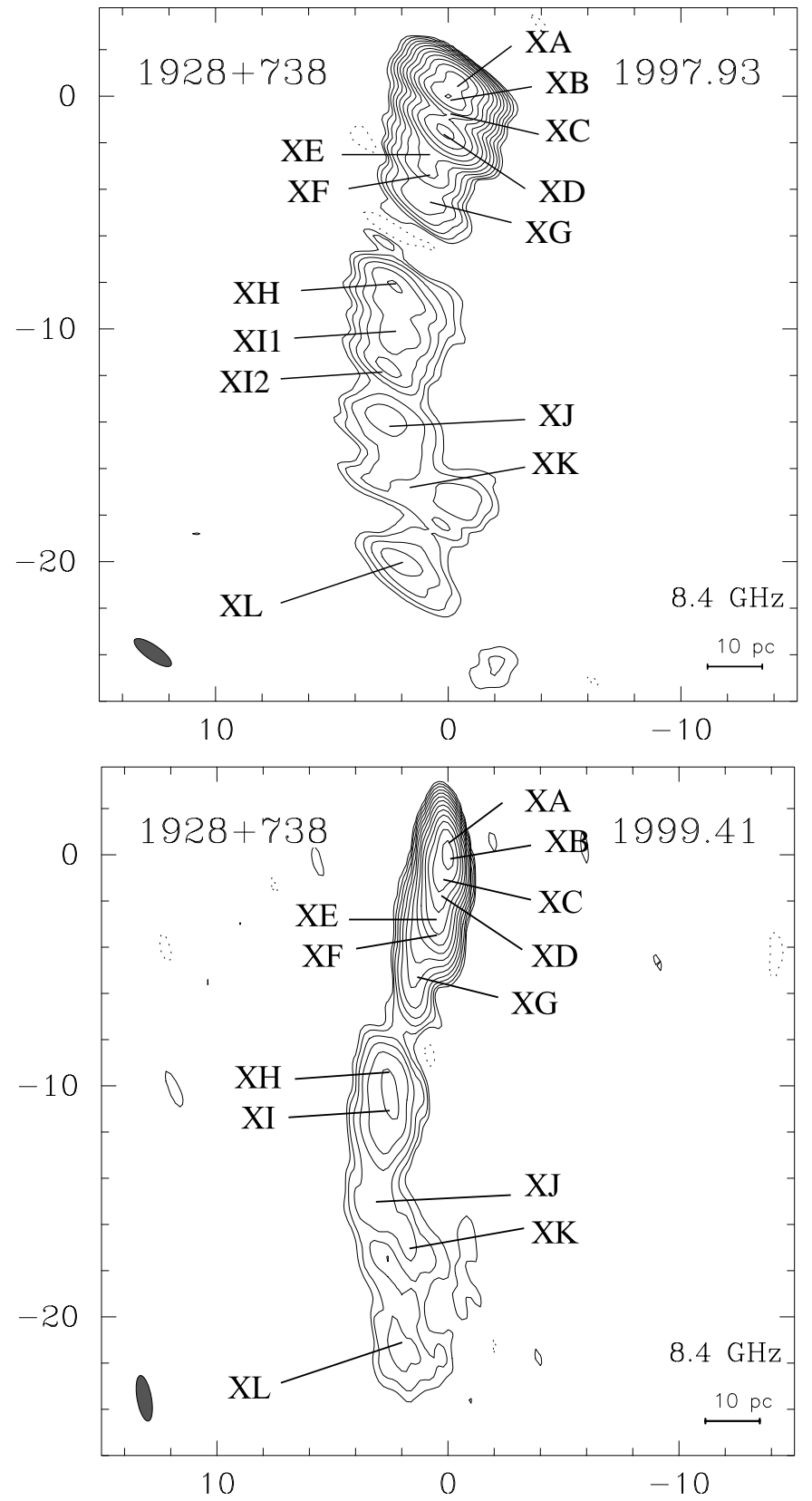

Fig. 14. VLBA images of QSO 1928+738 (4C 73.18), observed on 6 December 1997 (1997.93) and 28 May 1999 (1999.41). Axes are relative $\alpha$ and $\delta$ in mas. See Table 1 for contour levels, synthesized beam sizes (bottom left in the maps), and peak flux densities. See Table 2 for component parametrization.

Guirado et al. 1998; Ros et al. 1999) show emission west of the main component with the brightest features about 5-6 mas of the core, and emission extending out to 20 mas at $5 \mathrm{GHz}$ for epoch 1992.47 (Xu et al. 1993) and out to 40 mas at 2.3 GHz for epoch 1995.77 (Fey \& Charlot 1997). BL 2007+777 displays strong flux variability. Data from UMRAO at $8 \mathrm{GHz}$ show that the source changed the total flux density from $\sim 0.8$ Jy at 1996.5 to $\sim 1.8$ Jy at 1997.2 . 


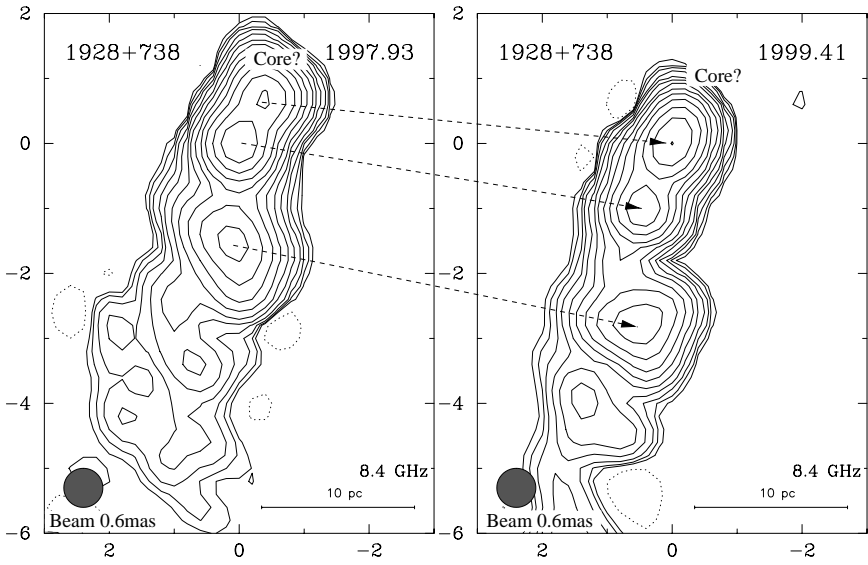

Fig. 15. VLBA images of QSO $1928+738$, convolved with a 0.6 mas circular beam. Contours are the same as in Fig. 14. The dashed lines represent a tentative association between jet regions from one epoch to another. Notice the structural changes in the maps, especially in the brighter regions. The images are centered in the brightest feature in both cases, which very probably diverges from a rigorous astrometric registration.

Throughout 1997 the source maintained a total flux density of $\sim 1.7 \mathrm{Jy}$ at $8.4 \mathrm{GHz}$, decreasing to $\sim 1.4$ Jy for our second epoch in mid 1999.

Our maps (Fig. 16) recover flux densities of 1.305 and $0.969 \mathrm{Jy}$ for the first and second epochs, respectively. Changes in the structure are evident. A secondary component about 2 mas (labeled $\mathrm{XC}+\mathrm{XD}$ in Fig. 16) west of the brightest feature (XA) appears at a different PA, having moved northwards from the first to the second epoch. This is confirmed by the $5 \mathrm{GHz}$ VLBI Space Orbiting Programme data from epochs close to ours (Krichbaum et al. 2000). A model fit with Gaussian components (Table 2 ) shows two components for the $\sim 2$ mas feature, $\mathrm{XC}$ and $\mathrm{XD}$, that change the $\mathrm{PA}$ from $-102^{\circ}$ and $-98^{\circ}$ in the first epoch to $-88^{\circ}$ and $-86^{\circ}$, respectively. Their distances from the brightest feature change from 1.2 and 1.6 mas to 1.4 and 1.7 mas, respectively. Assuming that component XA is stationary, these position changes correspond to northward superluminal motion (not only from the core outwards). The core area is double, with $\mathrm{XA}$ with similar flux densities in both epochs, and XB decaying to $30 \%$ of its previous flux densities in 1999.41 . Guirado et al. (2000) report a changing double structure in this core area at $43 \mathrm{GHz}$, which questions any registration of this region without astrometric information.

There are also structural changes in the components labeled XE and XF. While XE decays strongly in emission, XF becomes more prominent (it doubles its flux density from the first to the second epoch). This is consistent with space VLBI observations from Krichbaum et al. (2000), which report changes in the blobs of BL $2007+777$ within a month in the region 6-7 mas from the "core".

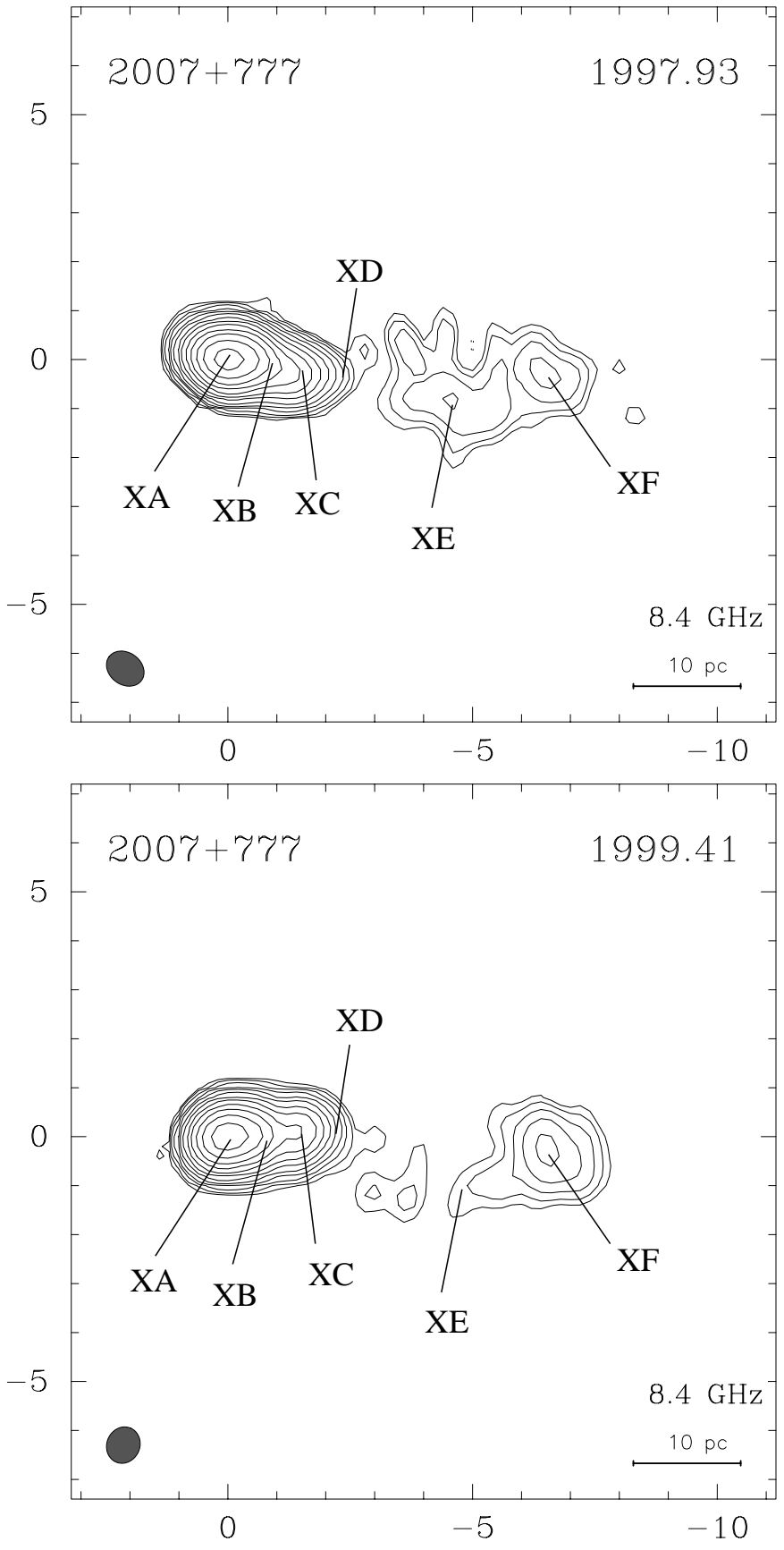

Fig. 16. VLBA images of BL $2007+777$, observed on 6 December 1997 (1997.93) and 28 May 1999 (1999.41). Axes are relative $\alpha$ and $\delta$ in mas. See Table 1 for contour levels, synthesized beam sizes (bottom left in the maps), and peak flux densities. See Table 2 for component parametrization.

The forthcoming exact alignment of the features provided by the astrometry should constrain the proper motions of the components of the jet of this radio source. 


\section{Summary}

We present $8.4 \mathrm{GHz}$ VLBA maps of the thirteen extragalactic radio sources from the S5 polar cap sample. For two epochs, all sources of the sample have been imaged (Figs. 2-16) with mas-resolution to the mJy level (Table 1). In all cases, we have modeled the interferometric visibilities with elliptical Gaussian models (Table 2).

We can report some morphological changes for selected radio sources. The sources with more evident variations are QSO 0016+731, QSO 0836+710, QSO 1928+738 and BL $2007+777$. For QSO 0016+731 the variations have been surprising, since the source is rather compact and does not show a prominent jet structure larger than 2-3 mas at $8.4 \mathrm{GHz}$. Its change in morphology is associated with the strong decrease in flux density of one of the components from the first to the second epoch. QSO 0836+710 and QSO 1928+738 show the familiar changes in structure produced by emerging components in a core-jet system, as reported by Otterbein et al. (1998) for the former, and Hummel et al. (1992a) for the latter. The BL Lac object $2007+777$ displays an interesting, and hard to explain change in position angle towards north for components near to the core, a fact also reported by Krichbaum et al. (2000) from $5 \mathrm{GHz}$ VSOP observations.

The images are the result of first and second epochs of a phase-delay astrometric program intended to check the absolute kinematics of all radio sources of the sample with precisions better than $100 \mu$ as. Such accurate results will be obtained after the mapping and astrometric reduction of more observing epochs is carried out. This multisource astrometric approach provides a large number of constraints for all the relative source pairs, which allow a precise registration of the maps through the observing epochs (as shown by Ros et al. 1999 for triplets of radio sources).

We have recently extended our astrometric programme to 15 and $43 \mathrm{GHz}$. At these frequencies, and based on test observations, we expect to attain astrometric precisions of 50 and $20 \mu \mathrm{as}$, respectively. The two-fold and five-fold improved resolution of those observations with respect to the observations presented here, combined with the expected astrometric precisions, will allow precise registrations of all the sources at each and all wavelengths for all epochs, and will provide unprecedented spectral information of components of milliarcsecond sources. The determination of the detailed kinematics and spectral content of the compact components of a complete sample of radio sources should then turn out to be a decisive element in our understanding of the activity around the cores of these objects and a definitive test of the standard jet model.

Acknowledgements. This work has been partially financed by Grant PB96-0782 of the Spanish DGICYT. This research has made use of data from the University of Michigan Radio Astronomy Observatory which is supported by the National
Science Foundation and by funds from the University of Michigan. NRAO is operated under license by Associated Universities Inc., under cooperative agreement with NSF.

\section{References}

Antonucci, R. R. J., Hickson, P., Olszewski, E. W., \& Miller, J. S. 1986, AJ, 92, 1

Appl, S., Sol, H., \& Vicente, L. 1996, A\&A, 310, 419

Bååth, L. B., \& Zhang, F. J. 1992, A\&A, 262, 1

Bartel, N., Herring, T. A., Ratner, M. I., Shapiro, I. I., \& Corey, B. E. 1986, Nature, 319, 733

Blandford, R. D., \& Königl, A. 1979, ApJ, 232, 34

Charlot, P. 1990, AJ, 99, 1309

Eckart, A., Witzel, A., Biermann, P., et al. 1985, ApJ, 296, 23

Eckart, A., Witzel, A., Biermann, P., et al. 1986, A\&A, 182, 17

Eckart, A., Witzel, A., Biermann, P., et al. 1987, A\&AS, 67, 121

Fey, A., \& Charlot, P. 1997, AJS, 111, 95

Fey, A., Clegg, A. W., \& Fomalont, E. B. 1996, AJS, 105, 299

Gabuzda, D. C., Cawthorne, T. V., Roberts, D. H., \& Wardle, J. F. C. 1992, AJ, 388, 40

Guirado, J. C., Marcaide, J. M., Elósegui, P., et al. 1995, A\&A, 293,613

Guirado, J. C., Marcaide, J. M., Alberdi, A., et al. 1995, AJ, 110,2586

Guirado, J. C., Marcaide, J. M., Ros, E., et al. 1998, A\&A, 336,385

Guirado, J. C., Marcaide, J. M., Pérez-Torres, M. A., \& Ros, E. 2000, A\&A, 353, L37

Hummel, C. A., Schalinski, C. J., Krichbaum, T. P., Witzel, A., \& Johnston, K. J. 1988, A\&A, 204, 68

Hummel, C. A., Schalinski, C. J., Krichbaum, T. P., et al. 1992a, A\&A, 257, 489

Hummel, C. A., Muxlow, T. W. B., Krichbaum, T. P., et al. 1992b, A\&A, 266, 93

Hummel, C. A., Krichbaum, T. P., Witzel, A., et al. 1997, A\&A, 324, 857

Johnston, K. J., Simon, R. S., Eckart, A., et al. 1987, ApJ, 313, 85

Kollgaard, R. I., Wardle, J. F. C., Roberts, D. H., \& Gabuzda, D. C. 1992, AJ, 104, 1687

Krichbaum, T. P., Jin, C., Kraus, A., Witzel, A., \& Zensus, J. A. 2000, Observations of Intraday Variable Sources, in Astrophysical Phenomena Revealed by Space VLBI, ed. H. Hirabayashi, P. G. Edwards, \& D. W. Murphy (Sagamihara, Japan: ISAS), 133

Krichbaum, T. P., Hummel, C. A., Quirrenbach, A., et al. 1990, A\&A, 230, 271

Krichbaum, T. P., Witzel, A., Graham, D. A., Schalinski, C. J., \& Zensus, J. A. 1993, New Results from VLBI at $43 \mathrm{GHz}$, in Sub-arcsecond Radio Astronomy, ed. R. J. Davis, \& R. S. Booth (Melbourne: Cambridge University Press), 181

Krichbaum, T. P., Witzel, A., Standke, K. J., et al. 1994, mmVLBI: Bending of Jets in the Vicinity of AGN, in Compact Extragalactic Radio sources, ed. J. A. Zensus, \& K. I. Kellermann (Green Bank, WV: NRAO), 39

Kühr, H., Witzel, A., Pauliny-Toth, I. I. K., \& Nauber, U. 1981, A\&AS, 45, 367 
Marcaide, J. M., \& Shapiro, I. I. 1983, AJ, 88, 1133

Marcaide, J. M., Elósegui, P., \& Shapiro, I. I. 1994, AJ, 108, 368

Murphy, D. W., Browne, I. W. A., \& Perley, R. A. 1993, MNRAS, 264, 298

O'Dea, C. P., Barvainis, R., \& Challis, P. M. 1988, AJ, 96, 435

Otterbein, K., Krichbaum, T. P., Kraus, A., et al. 1998, A\&A, 334,489

Pearson, T. J. 1991, BAAS, 23, 991

Pearson, T. J., \& Readhead, A. C. S. 1988, AJ, 328, 114

Peng, B., Kraus, A., Krichbaum, T. P., \& Witzel, A. 2000, A\&AS, 145,1

Pérez-Torres, M. A., Marcaide, J. M., Guirado, J. C., et al. 2000, A\&A, 360, 161

Perley, R. A. 1982, AJ, 87, 859

Perley, R. A. 1980, AJ, 85, 649

Quirrenbach, A., Witzel, A., Wagner, S., et al. 1991, ApJ, 372,71

Roos, N., Kaastra, J. S., \& Hummel, C. J. 1993, ApJ, 280, 542

Ros, E., Marcaide, J. M., Guirado, J. C., et al. 1999, A\&A, 348,381

Ros, E., Marcaide, J. M., Guirado, J. C., Sardón, E., \& Shapiro, I. I. 2000, A\&A, 356, 357
Rusk, R., \& Rusk, A. C. M. 1986, Canad. J. Phys., 64, 440

Russell, J. L., Johnston, K. J., Ma, C., Shaffer, D., \& de Vegt, C. 1991, AJ, 101, 2266

Schalinski, C. J. 1990, Dissertation, Untersuchungen der Zentralregionen aktiver Galaxienkerne (FriedrichWilhelms-Universität zu Bonn, Germany)

Shapiro, I. I., Wittels, J. J., Counselman, C. C., et al. 1979, AJ, 84, 1459

Shepherd, M. C., Pearson, T. J., \& Taylor, G. B. 1994, BAAS, 26, 987

Steffen, W. 1994, Dissertation, Helikale Strukturen in aktiven Galaxienkernen (Friedrichs-Wilhelms-Universität zu Bonn, Germany)

Stickel, M., \& Kühr, H. 1996, A\&AS, 115, 1

Strom, R. G., \& Biermann, P. L. 1991, A\&A, 242, 313

Wagner, S. J., Witzel, A., Heidt, J., et al. 1996, AJ, 111, 2187

Witzel, A., Schalinski, C.J., Johnston, K. J., et al. 1988, A\&A, 206, 245

Xu, W., Readhead, A. C. S., Pearson, T. J., Polatidis, A. G., \& Wilkinson, P. N. 1995, ApJS, 99, 297 